INRA Prod. Anim., 2008, 21 (2), 159-166

\title{
Approches combinées de génomique positionnelle et expressionnelle pour l'étude des gènes contrôlant la qualité de la viande chez les volailles
}

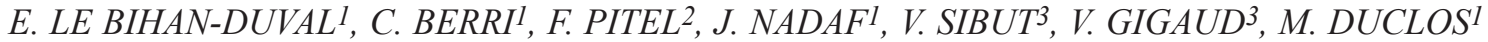 \\ IINRA, UR83 Recherches Avicoles, F-37380 Nouzilly, France \\ 2 INRA, ENVT, UMR444 Génétique cellulaire, F-31326 Castanet-Tolosan, France \\ ${ }^{3}$ Institut Technique Avicole, Unité de Recherches Avicoles, F-37380 Nouzilly, France \\ mailto : Elisabeth.Lebihan@tours.inra.fr
}

\begin{abstract}
Satisfaire aux exigences de qualité, de conservation et de transformation, est devenu un enjeu majeur en production de viande de volailles. Des études génomiques sont aujourd'hui en cours pour identifier les gènes, ou plus largement les voies métaboliques, impliqués dans la qualité. A l'avenir, ces outils moléculaires pourront contribuer au développement de stratégies génétiques et d'élevage optimisant la qualité de la viande.
\end{abstract}

Depuis les années 60, la filière avicole s'est considérablement développée, grâce aux progrès apportés par l'alimentation, la rationalisation des modes d'élevage, la médecine vétérinaire ainsi que l'amélioration génétique des animaux (Beaumont et al 2004). Chez les volailles de type chair, des progrès très importants ont été réalisés sur les performances de croissance, la composition corporelle ou encore l'efficacité alimentaire, l'ensemble ayant fortement réduit les coûts de production des animaux. Mais, après des décennies de développement, les données économiques sont aujourd'hui moins favorables puisque, entre 1998 et 2005, une baisse de $17 \%$ de la production française de volailles a été observée (Magdelaine 2006). Dans le même temps, le marché national s'est largement diversifié, offrant des perspectives de développement sur le segment des produits élaborés. Entre 2005 et 2010 , leur part de marché devrait passer pour le poulet de $23 \%$ à $28 \%$ de la viande consommée (Magdelaine 2006).

Du fait des évolutions des modes de consommation, les exigences sur la qualité de la viande se sont faites plus insistantes, à la fois pour satisfaire les besoins des consommateurs et augmenter le niveau de compétitivité des filières françaises et plus largement euro- péennes. Plusieurs composantes de la qualité de la viande, tel que le Pouvoir de Rétention en Eau (PRE), la texture ou la couleur, sont influencées par le métabolisme musculaire et en particulier la cinétique de chute du $\mathrm{pH}$ post mortem (Berri 2000). Ainsi, les viandes à $\mathrm{pH}$ ultime $(\mathrm{pHu})$ bas (inférieur à 5,7$)$ ou à vitesse de chute de $\mathrm{pH}$ élevée $(\mathrm{pH}$ inférieur à 6,2 à 15 min post mortem) présentent un faible PRE et une texture dure et sèche après cuisson (Barbut 1997). Par abus de langage, elles sont parfois qualifiées de PSE (Pale, Soft, Exudative), par référence aux défauts du même type décrits chez le Porc. Les viandes à haut $\mathrm{pH}$ (supérieur à 6,0$)$ sont peu adaptées à la conservation en cru en raison d'un développement microbien favorisé (Allen et al 1997) et d'une dégradation de la jutosité. Les données récemment collectées en abattoir sur du filet de poulet soulignent la très forte variabilité des valeurs du $\mathrm{pH}$ (Gigaud et al 2007, figure 1), à l'origine d'une forte hétérogénéité de la qualité du pro-

Figure 1. Variabilité du pH ultime du filet chez le poulet standard abattu en conditions industrielles (minima, maxima et moyennes par lot, avec 250 animaux mesurés pour chacun des 12 lots) (Gigaud et al 2007).

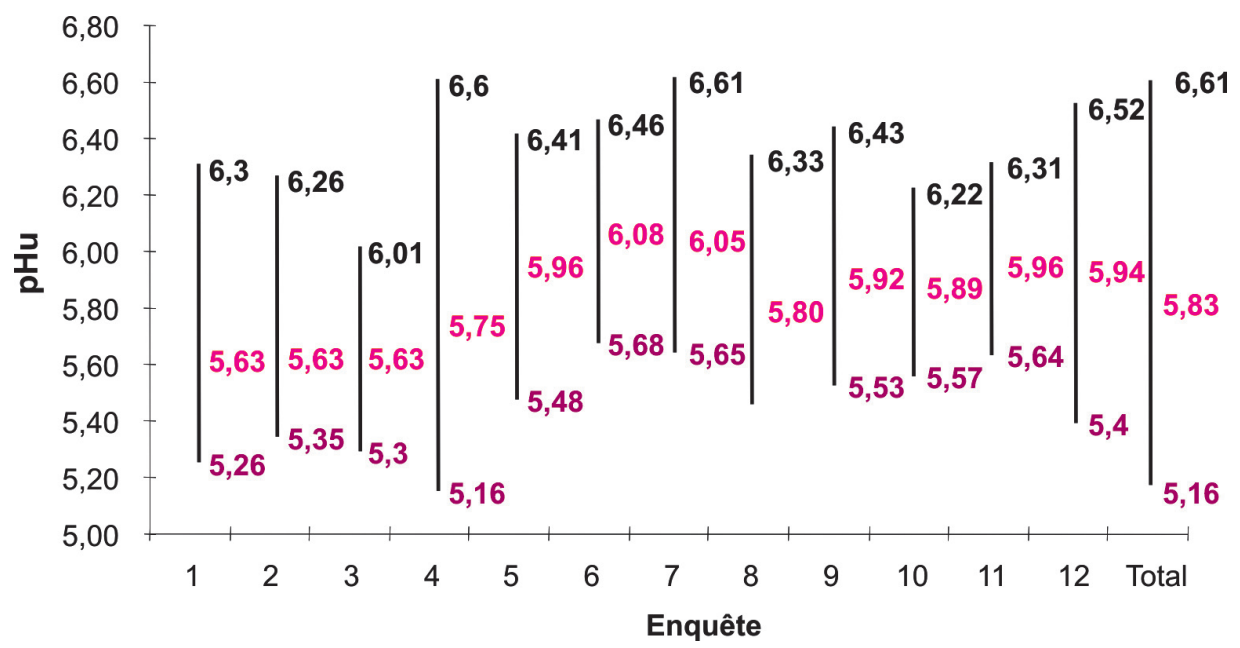


duit final (viande de découpe ou produit élaboré). Pour remédier à cette hétérogénéité, des adaptations technologiques tel qu'un tri en ligne de la viande sur sa couleur sont aujourd'hui envisagées (Popot et al 2006). Néanmoins, des gains substantiels pourraient aussi être réalisés par une amélioration en amont de la qualité de la matière première.

Le métabolisme musculaire post mortem et la qualité de la viande dépendent de nombreux paramètres, tels que les caractéristiques intrinsèques de l'animal ou ses conditions d'élevage et d'abattage (Berri 2000, Berri et al 2005, Debut et al 2003, 2004, 2005). Les données disponibles au niveau génétique montrent qu'une sélection sur les caractéristiques de qualité ( $\mathrm{pH}$, couleur, PRE, texture...) est envisageable (Le Bihan-Duval et al 2001, 2008). Toutefois, sa mise en œuvre est aujourd'hui freinée par la nécessité d'abattre les animaux pour évaluer la qualité, ce qui implique des coûts importants et une diminution de l'efficacité de sélection. La Sélection Assistée par Marqueurs (SAM) est donc une voie à explorer si l'on veut accroître les efforts de sélection sur la qualité. Cette sélection pourrait en premier lieu bénéficier aux souches de type standard, largement utilisées pour les marchés de la découpe et des produits élaborés. A terme, elle pourrait aussi intéresser les filières alternatives de type «Label Rouge» et «certifié», qui sont aujourd'hui largement tournées vers la production du poulet entier (avec plus de 65\% des parts de marché), mais qui devront à terme s'adapter aux nouveaux débouchés de la découpe et des produits élaborés.

\section{1 / Les modèles d'études : apport des lignées expéri- mentales et commerciales}

En aviculture, la diversité des modes de production s'appuie sur une diversité des génotypes qui présentent des performances de croissance et de composition corporelle bien démarquées. Ainsi, s'il faut aujourd'hui moins de 6 semaines à un poulet de type «standard» pour atteindre le poids requis par le marché (environ $2,5 \mathrm{~kg}$ ), les souches de type label à croissance lente l'atteignent avec une durée minimale d'élevage de 81 jours (fixée par la notice technique Label Rouge). Des variations de composition corporelle existent aussi, certaines souches standard ayant été sélectionnées pour une augmentation du rendement en filet (morceau noble de la carcasse) et une diminution de l'adiposité.

Les études menées au sein de lignées expérimentales ou commerciales de poulet montrent que les caractéristiques musculaires et la qualité de la viande du filet varient en fonction des caractéristiques de croissance et de composition corporelle des animaux. Ainsi, chez des poulets commerciaux à croissance rapide, un fort développement musculaire est associé à une hypertrophie des fibres, et s'accompagne d'une diminution des réserves du muscle en glycogène (Berri et al 2007). Au sein de ce génotype, le poids du muscle du filet est en effet génétiquement négativement relié à son Potentiel Glycolytique (PG) $(-0,58)$, et positivement à son $\mathrm{pH}$ ultime $(0,84)$ (Le Bihan-Duval et al 2008). Dans ce type de lignée, accrô̂tre le potentiel génétique pour le déve- loppement musculaire conduit donc à un filet moins acide, et en conséquence moins pâle, moins exsudatif et plus tendre. La comparaison de deux lignées expérimentales de poulets, sélectionnées de façon divergente sur l'engraissement abdominal (Leclercq et al 1980), souligne également un lien entre adiposité et qualité (tableau 1). Le génotype «maigre» présente des réserves en glycogène dans le filet plus faibles, à l'origine d'un $\mathrm{pH}$ ultime plus élevé que le génotype gras $(5,8 v s 5,6)$. Une corrélation négative (de - 0.52 ) entre engraissement abdominal et $\mathrm{pHu}$ a également été observée au niveau génétique au sein d'une autre lignée expérimentale de poulet (Le BihanDuval et al 2001).

La sélection sur la vitesse de croissance a aussi modifié les caractéristiques musculaires et de qualité de la viande chez le Poulet. C'est ce qu'illustre l'étude des lignées «lourde» et «légère» sélectionnées à l'INRA (Ricard 1975), extrêmement différentes tant sur le plan du poids vif (le critère de sélection appliqué) que de leur composition corporelle (tableau 1). Dans ce modèle expérimental, la sélection pour une augmentation du poids vif a entrấné une vitesse de chute du $\mathrm{pH}$ plus importante (révélée par un $\mathrm{pH}$ à $15 \mathrm{~min}$ post mortem plus faible, 6,2 vs 6,3), un $\mathrm{pH}$ ultime plus bas $(5,7$ vs 6,1$)$ et une moindre coloration (dans les teintes rouge et jaune) de la viande du filet. Ces variations du métabolisme post mortem et de la qualité semblent en partie expliquées par celles des réserves du muscle en glycogène disponibles au moment de l'abattage, et par les variations d'activité physique des oiseaux sur la chaîne d'abattage. Ainsi, le génotype à croissance «rapide» pré-

Tableau 1. Poids vif, composition corporelle et caractéristiques de qualité de la viande (moyenne \pm écart-type) dans le filet des poulets légers à croissance lente ou lourds à croissance rapide, et maigres ou gras abattus à l'âge de 9 semaines.

\begin{tabular}{|c|c|c|c|c|c|c|}
\hline Caractères & $\begin{array}{l}\text { Lignée } \\
\text { légère } \\
(n=56)\end{array}$ & $\begin{array}{l}\text { Lignée } \\
\text { lourde } \\
(n=53)\end{array}$ & $\begin{array}{l}\text { Effet } \\
\text { lignée }\end{array}$ & $\begin{array}{c}\text { Lignée } \\
\text { maigre } \\
(n=60)\end{array}$ & $\begin{array}{l}\text { Lignée } \\
\text { grasse } \\
(n=60)\end{array}$ & $\begin{array}{l}\text { Effet } \\
\text { lignée }\end{array}$ \\
\hline \multicolumn{7}{|l|}{ Croissance corporelle } \\
\hline Poids vif $(\mathrm{g})$ & $683 \pm 67$ & $1922 \pm 157$ & $<.0001$ & $2522 \pm 193$ & $2627 \pm 162$ & $<.001$ \\
\hline Gras abdominal (\%) & $0,2 \pm 0,2$ & $2,5 \pm 0,7$ & $<.0001$ & $1,4 \pm 0,5$ & $3,9 \pm 0,7$ & $<.001$ \\
\hline Filet (\%) & $10,4 \pm 0,8$ & $11,4 \pm 0,8$ & $<.0001$ & $12,8 \pm 0,9$ & $11,5 \pm 0,9$ & $<.001$ \\
\hline Cuisse (\%) & $22,0 \pm 0,7$ & $23,2 \pm 0,9$ & $<.0001$ & NA & NA & NA \\
\hline \multicolumn{7}{|l|}{ Indicateurs de qualité } \\
\hline Luminosité $\left(\mathrm{L}^{*}\right)$ & $45,6 \pm 1,8$ & $48,3 \pm 3,2$ & $<.0001$ & $44,9 \pm 2,6$ & $47,4 \pm 2,7$ & $<.001$ \\
\hline Indice de rouge $\left(a^{*}\right)$ & $1,6 \pm 0,7$ & $-0,2 \pm 0,8$ & $<.0001$ & $-0,3 \pm 0,7$ & $-1,0 \pm 0,7$ & $<.001$ \\
\hline Indice de jaune $\left(b^{*}\right)$ & $13,3 \pm 1,4$ & $9,4 \pm 1,2$ & $<.0001$ & $9,3 \pm 1,0$ & $8,3 \pm 1,3$ & $<.001$ \\
\hline $\mathrm{pH} 15 \min$ & $6,33 \pm 0,16$ & $6,20 \pm 0,22$ & 0.0004 & $6,38 \pm 0,21$ & $6,36 \pm 0,22$ & NS \\
\hline $\mathrm{pH}$ ultime & $6,14 \pm 0,14$ & $5,74 \pm 0,09$ & $<.0001$ & $5,79 \pm 0,12$ & $5,66 \pm 0,11$ & $<.001$ \\
\hline Pertes en eau $(\%)$ & $2,1 \pm 1,5$ & $2,3 \pm 1,2$ & NS & $1,1 \pm 0,6$ & $1,4 \pm 0.6$ & $<.05$ \\
\hline
\end{tabular}

$\mathrm{NS}=$ non significatif $; \mathrm{NA}=$ données manquantes 
sente un potentiel glycolytique dans le filet plus important et une activité physique plus soutenue, conduisant à une acidification du muscle à la fois plus rapide et plus prononcée.

Les données acquises sur ces modèles expérimentaux et commerciaux soulignent donc la grande plasticité des volailles et attirent l'attention sur les conséquences possibles, au niveau du muscle et de la qualité, de la sélection menée dans les lignées. Largement complémentaires, ces modèles permettent de développer efficacement les études de génomique sur la qualité de la viande de volailles. A terme, celles-ci aboutiront à une meilleure compréhension des mécanismes moléculaires reliant croissance et qualité et, nous l'espérons, au développement de stratégies génétiques et d'élevage visant à l'amélioration conjointe de ces caractères.

\section{2 / Apport de la génomique positionnelle : détection de QTL}

Le développement des techniques de biologie moléculaire a permis dans de nombreuses espèces d'intérêt agronomique la construction de cartes génétiques, organisées en groupes de liaisons, rassemblant eux-mêmes plusieurs marqueurs servant de «balises» sur le génome. Ces marqueurs génétiques, fragments d'ADN, sont polymorphes c'est-à-dire qu'ils présentent plusieurs formes ou «allèles». Les marqueurs les plus utilisés ces dernières années ont été les microsatellites, dont le polymorphisme correspond à des nombres variables de répétitions de motifs courts (2 à $4 \mathrm{pb}$ ) sur l'ADN. Nous en comptions environ 800 sur la carte consensus publiée en 2000 (Groenen et al 2000). Depuis la publication de l'assemblage de la séquence, des marqueurs de ce type peuvent être développés «in silico» dans toute région du génome intéressante (http://genome.

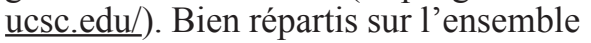
du génome, ils ont été largement utilisés pour l'identification des régions chromosomiques expliquant une part significative de la variabilité des caractères d'intérêt ou «QTL» (Quantitative Trait Loci). Comme l'illustre la figure 2, la détection d'un QTL repose sur la comparaison, au sein des familles, de la performance moyenne du caractère (ou phénotype) entre les deux groupes de descendants ayant reçu un allèle (M1) ou l'autre (M2) de
Figure 2. Principe général de détection d'un QTL influençant le caractère d'intérêt à partir de l'information au marqueur M (d'après Le Roy 2001).
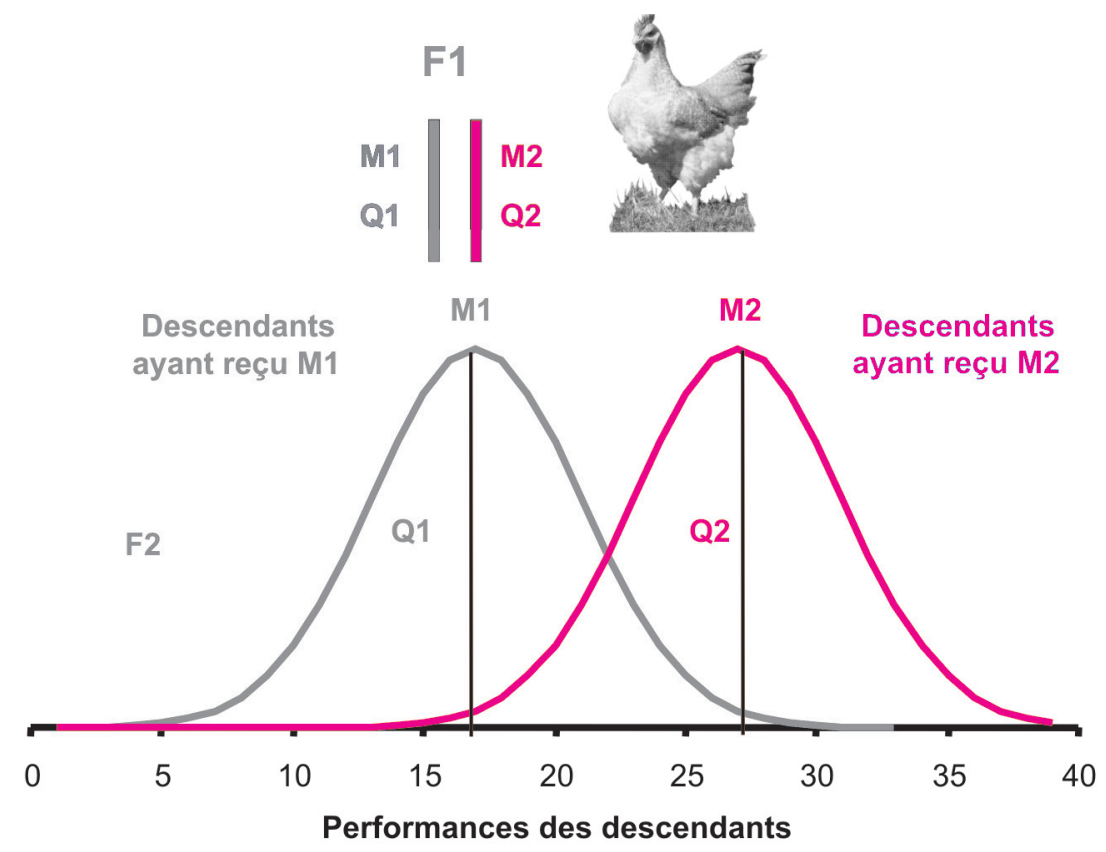

leur père (ou mère) hétérozygote au marqueur (F1). Si cette différence existe, on peut supposer que le marqueur $M$ est transmis physiquement en même temps qu'un gène $\mathrm{Q}$ affectant le caractère (Le Roy 2001). Les statistiques de test (par régression ou Maximum de Vraisemblance; Kao 2000, Elsen 2001) permettent de comparer l'hypothèse $\mathrm{H} 1$ de ségrégation d'un QTL en une position donnée sur le génome versus l'hypothèse H0 d'absence de QTL. Si le QTL est confirmé, il indique la présence dans cette région chromosomique d'un ou plusieurs gènes dont les différentes formes sont à l'origine de la variabilité du caractère étudié.

Si chez le poulet de nombreux QTLs ont déjà été identifiés pour les caractères de production (Hocking 2005, Abasht et al 2006, Chicken QTL database : http://www.animalgenome.org/ QTLdb/chicken.html), la qualité de la viande a fait l'objet d'un nombre très réduit d'études. Initié en 2001 en collaboration entre l'INRA et des équipes américaines, le projet IFAFS «A Consortium for Functional Mapping of Growth-Regulating Genes in Broiler Chickens» a permis de développer les études sur la qualité (en relation avec la croissance et la composition corporelle des animaux), en s'appuyant sur les souches «lourde» ou «légère» et «maigre» ou «grasse» sélectionnées à l'INRA. Pour chacun de ces modèles, un croisement de seconde génération de près de 700 animaux F2 a été caractérisé pour la croissance et la qualité et l'ensemble des animaux génotypé pour environ 120 marqueurs microsatellites, répartis de manière aussi uniforme que possible sur le génome. La qualité du filet a été évaluée par les mesures précoce et finale du pH (à 15 min et $24 \mathrm{~h}$ post mortem), la luminance ( $\left.\mathrm{L}^{*}\right)$, les indices de rouge $(\mathrm{a} *)$ et de jaune $(\mathrm{b} *)$, et les pertes en eau par exsudation. L'analyse du croisement entre lignées lourde et légère a permis d'identifier plusieurs QTL, contrôlant d'une part les caractéristiques de croissance ou de composition corporelle et d'autre part la qualité de la viande. Au vu des premiers résultats, il s'agit plutôt de régions distinctes, ce qui laisse penser qu'en exploitant ces QTL on pourrait faire varier la qualité sans forcément modifier la croissance. Un QTL hautement significatif (figure 3) a ainsi été identifié sur le chromosome 11 pour la couleur, l'un des caractères déterminants pour la qualité de la viande. Deux régions significatives pour le $\mathrm{pH} 15$ ont aussi été détectées sur les chromosomes 1 et 2 , à proximité pour la première d'un QTL affectant les pertes en eau par exsudation (Nadaf et al 2007).

Néanmoins, comme pour la majorité des autres caractères (Hocking 2005), la localisation de ces QTL pour la qualité reste très imprécise : plusieurs dizaines de centimorgans (de 20 à 50), représentant plusieurs centaines de gènes candidats positionnels. Cette faible résolution du QTL induit à terme des pertes d'efficacité de la SAM, du fait de recombinaisons possibles entre les marqueurs et le QTL. En conséquence et du fait des caractéristiques 
Figure 3. Profil de vraisemblance (Likelihood Ratio Test, LRT) et seuil de signification à 1\% du QTL de couleur jaune (supérieure) et rouge (courbe inférieure) sur le chromosome 11 (Nadaf et al 2007).

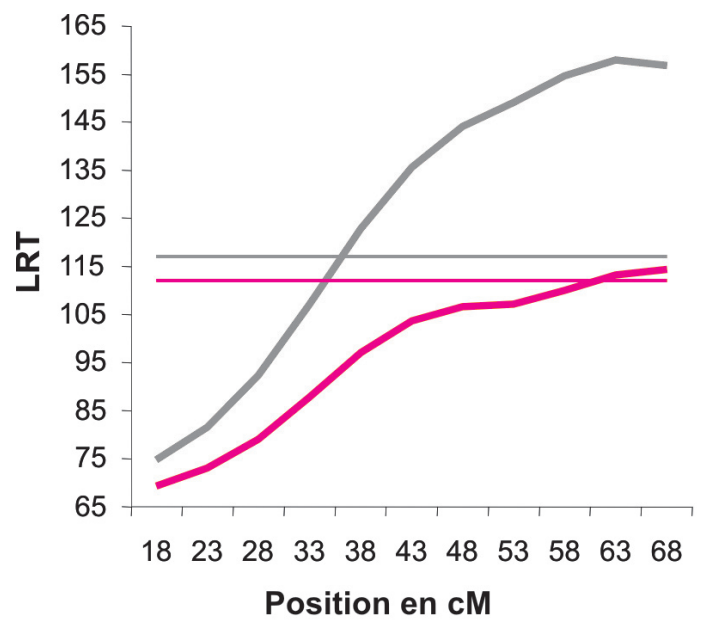

propres des schémas avicoles (cycles de reproduction courts et familles de relative petite taille), il semble aujourd'hui nécessaire pour les applications en sélection d'affiner les régions QTL pour si possible identifier les polymorphismes sous-jacents, ou à défaut des marqueurs génétiques qui leur soient étroitement associés. Coproduits du séquençage du génome de la poule (International Chicken Genome Sequencing Consortium, 2004), les marqueurs de type SNP (Single Nucleotide Polymorphism, mutation d'une base au sein d'un fragment d'ADN), ont l'avantage d'être à la fois très nombreux (un SNP tous les 200 paires de bases environ, Wong et al 2004) et d'un coût de typage réduit. L'accès à cette nouvelle technologie de marqueurs pour le poulet devrait donc à court terme permettre une détection à la fois plus systématique et plus précise des QTL contrôlant les caractères d'intérêt (dont la qualité) et faciliter l'identification des gènes candidats.

L'approche «gène candidat» pour de la sélection implique d'identifier des variations au sein (ou à proximité) de la séquence du gène et d'étudier l'association entre les différents allèles de ce gène et le caractère d'intérêt. Outre les gènes candidats suggérés par les résultats QTL dans l'espèce d'intérêt, cette approche peut aussi tirer profit des connaissances acquises dans d'autres espèces, où des gènes majeurs ont déjà été identifiés. Ainsi pour la qualité, on sait que les chutes de $\mathrm{pH}$ rapides parfois observées chez le poulet ou la dinde présentent des similarités avec le syndrome de type PSE observé chez le Porc. Celui-ci est du à une mutation (de type $\mathrm{C} / \mathrm{T}$ ) dans le gène codant pour la protéine musculaire RYR1 (canal cal- cique également dénommé récepteur à la ryanodine). Chez le poulet, deux isoformes (RYR1 et RYR3) de ces récepteurs ont été identifiés (Ottini et al 1996) et leurs gènes respectifs sont positionnés. Le développement de marqueurs moléculaires à proximité ou au sein de ces deux gènes devrait permettre de préciser leurs effets sur la cinétique de chute du $\mathrm{pH}$, mesurée dans différentes populations de poulets. De façon analogue, l'effet du gène de l'AMP kinase PRKAG3 (ou gène RN pour Rendement Napole), responsable chez le Porc d'un excès de glycogène musculaire à l'origine des problèmes de viande acide (Milan et al 2000), reste à étudier chez le poulet. A terme, l'ensemble de ces approches devrait permettre le développement de tests génétiques (portant idéalement sur les mutations causales), applicables à grande échelle pour le typage des animaux et l'évaluation de leur potentiel génétique pour la qualité.

\section{3 / Apport de la génomique expressionnelle : études de transcriptome}

Le développement récent d'outils d'analyse à grande échelle du niveau d'expression des gènes a permis dans les espèces d'intérêt agronomique, dont le poulet, la mise en oeuvre d'approches généralistes pour l'étude des gènes associés aux variations des phénotypes d'intérêt, dont la qualité. Des puces à ADN (ou microarrays) portant l'empreinte d'environ 14000 gènes uniques ont été développées à l'Université du Delaware (Cogburn et al 2004) dans le cadre du projet IFAFS. Elles ont permis d'engager un pro- gramme de comparaison du transcriptome (niveaux des messagers) musculaire chez les animaux des lignées INRA maigre ou grasse et lourde ou légère. Dans ces dernières, plusieurs gènes sont différentiellement exprimés entre 1 et 11 semaines d'âge, et ce de façon cohérente. Des catégories très variées, comme le gène Pr57 (codant pour une protéine d'enveloppe rétrovirale) ou l'alpha-énolase (enzyme impliquée dans la voie de la glycolyse), peuvent être concernées (Jenkins et al 2007). L'effet de ces gènes candidats sur les variations de croissance musculaire et de qualité devra bien sûr être validé au sein de ces lignées sur de plus larges effectifs, puis dans d'autres populations en particulier commerciales. Déjà, de nouvelles technologies de biopuces apparaissent avec le passage chez le poulet aux puces oligonucléotides (ARK genomics ; CRB-GADIE à l'INRA), plus sensibles et contenant un plus grand nombre de gènes (environ 20000 gènes uniques). Celles-ci bénéficieront aux travaux en cours sur le transcriptome musculaire d'animaux à «fort» ou «faible» potentiel glycolytique, issus des lignées «maigre» et «grasse» ou d'une population commerciale présentant une large variabilité pour ce paramètre de qualité (Projet QualViVol, ANR-Genanimal et Agenavi).

Les études de transcriptome et les recherches de QTL vont générer des listes de gènes candidats, qu'il faudra confronter afin d'identifier des «doubles» candidats à la fois expressionnels et positionnels. Les banques de données généralistes, spécialisées, ou d'annotations (telle que GeneOntology) peuvent fournir des informations utiles sur la fonction des gènes, permettant de relier certains candidats aux phénotypes d'intérêt. Récemment, d'autres outils ont été développés pour faciliter l'étude des voies de régulation, en réalisant des analyses in silico. Comme l'illustre la figure 4, le logiciel «Genomatix» (http://www.genomatix. de/company/people.html) permet par exemple d'obtenir une représentation graphique, sous forme de réseaux, de l'ensemble des gènes d'une liste de départ (par exemple les gènes candidats positionnels localisés au sein d'une région QTL) et de gènes co-cités dans la littérature. Les réseaux obtenus peuvent ainsi révéler des liens éventuels entre candidats positionnels et expressionnels, permettre de reconstruire des cascades de régulation, et faciliter in fine l'identification du gène causal. 
Figure 4. Représentation des gènes candidats inclus dans la liste d'entrée (par exemple le gène CBFA2T3) et des gènes co-cités dans la littérature (BiblioSphère de Génomatix).

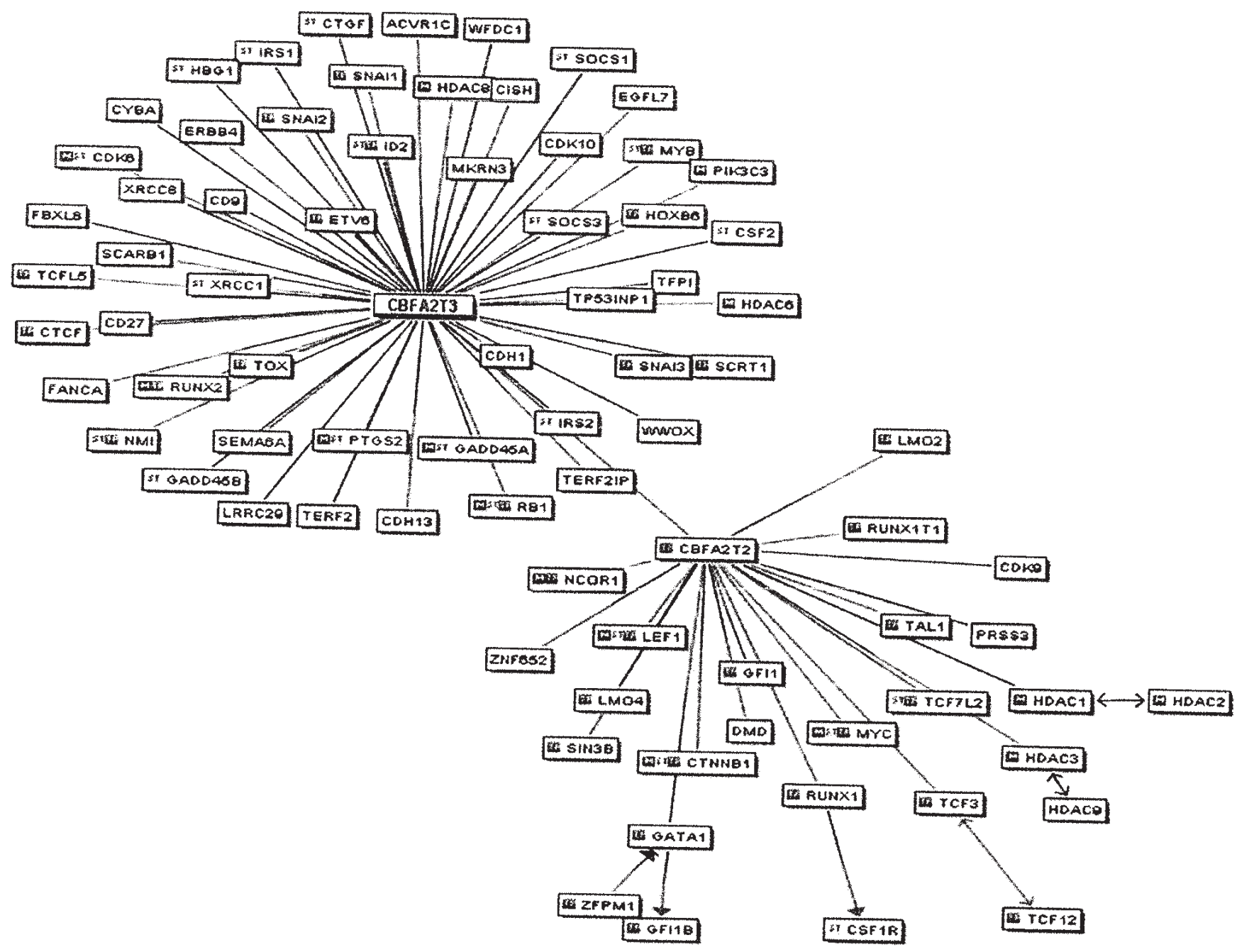

Une caractérisation fonctionnelle des QTL impliqués dans les variations de qualité de la viande peut aussi être envisagée par une approche expérimentale de type «eQTL». Si l'on réserve le terme de QTL à des régions responsables de la variabilité d'un caractère quantitatif (par exemple un indicateur de qualité de viande), la notion de eQTL se rapporte à la détection de régions contrôlant la variabilité d'expression d'un gène. Cette démarche a déjà été appliquée avec succès chez la levure (Brem et al 2002) et la souris (Schadt et al 2003). Elle est aujourd'hui en cours d'expérimentation chez le poulet dans des études sur le contrôle de la croissance et l'engraissement (projet ANR - Genanimal eQTL, 2004) et de la qualité des viandes (projet QualViVol). En pratique, la démarche retenue pour la qualité des viandes s'intéressera plus particulièrement aux gènes impliqués dans les variations du PG musculaire et donc du $\mathrm{pH}$ ultime de la viande. Elle consistera à mesurer par RT-PCR quantitative l'expression de gènes candidats fonctionnels (choisis en fonction des connaissances sur les voies métaboliques impliquées) ou expressionnels (issus des études du transcriptome) chez des animaux extrêmes de dispositifs familiaux. La colocalisation de QTL contrôlant les paramètres de qualité ( $\mathrm{PG}$ et $\mathrm{pHu}$ ) et eQTL contrôlant l'expression des gènes devrait permettre d'identifier des voies de régulation et d'avancer dans la recherche du ou des gènes causaux. Cette approche permettra également d'apprécier dans quelle mesure travailler sur des mesures d'expressions géniques pourrait, du fait d'une meilleure caractérisation du phénotype d'intérêt (croissance ou qualité), aboutir à une localisation plus précise des QTL.

\section{Conclusions}

Les recherches menées pour identifier les gènes impliqués dans la qualité des viandes de volailles bénéficient comme pour les autres caractères des progrès considérables réalisés ces dernières années dans le domaine de la biologie à haut débit. Elles impliquent des approches multidisciplinaires, pour couvrir des champs de compétences variées en génétique, physiologie, bioinformatique, etc. Les connaissances acquises sur les modèles animaux restent un point crucial pour la réussite de ces recherches, car elles permettent de choisir des conditions d'études optimales et facilitent l'identification des voies métaboliques et des gènes impliqués. Enfin, ces recherches impliquent un partenariat actif avec les acteurs de la filière, pour faciliter le transfert des résultats vers la sélection et la production des volailles de chair. 


\section{Références}

Abasht B., Dekkers J.C.M., Lamont S.J., 2006. Review of Quantitative Trait Loci identified in the chicken. Poult. Sci., 85, 2079-2096.

Allen C.D., Russell S.M., Fletcher D.L., 1997. The relationship of broiler breast meat color and $\mathrm{pH}$ to shelf-life and odor development. Poult. Sci., 76, 1042-1046.

Barbut S., 1997. Problem of pale soft exudative meat in broiler chickens. Br. Poult. Sci., 38, 355-358.

Beaumont C., Le Bihan-Duval E., Juin H., Magdelaine P., 2004. Productivité et qualité du poulet de chair. INRA Prod. Anim., 17, 265-273.

Berri C., 2000. Variability of sensory and processing qualities of poultry meat. World's Poult. Sci. J., 56, 209-224.

Berri C., Debut M., Santé-Lhoutellier V., Arnould C., Boutten B., Sellier N., Baéza E., Jehl N., Jégo Y., Duclos M.J., Le Bihan-Duval E., 2005. Variations in chicken breast meat quality: a strong implication of struggle and muscle glycogen level at death. Br. Poult. Sci., 46, 572579.

Berri C., Le Bihan-Duval E., Debut M., SantéLhoutellier V., Baéza E., Gigaud V., Jégo Y., Duclos M.J., 2007. Consequence of muscle hypertrophy on Pectoralis major characteristics and breast meat quality of broiler chickens. J. Anim. Sci., 85, 2005-2011.

Brem R.B., Yvert G., Clinton R., Kruglyak L., 2002. Genetic dissection of transcriptional regulation in budding yeast. Science, 296, 752-755.

Cogburn L.A., Wang X., Carre W., Rejto L., Aggrey S.E., Duclos M.J., Simon J., Porter T.E., 2004. Functional genomics in chickens: development of integrated-systems microarrays for transcriptional profiling and discovery of regulatory pathways. Comp. Funct. Genomics, 5, 253-261.

Debut M., Berri C., Baéza E., Sellier N., Arnould C., Guémené D., Jehl N., Boutten B., Jégo Y., Beaumont C., Le Bihan-Duval E., 2003. Variation of chicken technological meat quality in relation to genotype and preslaughter stress conditions. Poult. Sci., 82, 1829-1838.

Debut M., Le Bihan-Duval E., Berri C., 2004. Impacts des conditions de pré-abattage sur la qualité technologique de la viande de volaille. Sci. Tech. Avicoles, 48, 4-13.

Debut M., Berri C., Arnould C., Guémené D., Santé-Lhoutellier V., Sellier N., Baéza E., Jehl N., Jégo Y., Beaumont C., Le Bihan-Duval E.,
2005. Behavioural and physiological responses of three chicken breeds to pre-slaughter shackling and acute heat stress. Brit. Poult. Sci., 46, 527-535

Elsen J.M., 2001. Méthodes de détection : approche multiQTL. In : QTL - De la détection à l'utilisation. Séminaire du département de Génétique Animale de l'INRA. 24-26 Septembre, Batz-sur-Mer, France, 11-21.

Gigaud V., Geffrard A., Berri C., Le BihanDuval E., Travel A., Bordeau T., 2007. Conditions environnementales ante mortem (ramassage - transport - abattage) et qualité technologique. Viandes et Produits Carnés, 26, 1720.

Groenen M.A., Cheng H.H., Bumstead N., Benkel B.F., Briles W.E., Burke T., Burt D.W., Crittenden L.B., Dodgson J., Hillel J., Lamont S., Ponce de Leon A., Soller M., Takahashi H., Vignal A., 2000. A Consensus Linkage Map of the Chicken Genome. Genome Res., 10, 137147.

Hocking P.M., 2005. Review of QTL mapping in chickens. World's Poultr. Sci. J., 61, 215-226.

Jenkins C., Le Bihan-Duval E., Berri C., Simon J., Cogburn L., Duclos M.J., 2007. Comparaison des transcriptomes musculaires de deux génotypes de poulet lourds et légers. 7 emes Journ. Rech. Avicole, Tours, France, 28-29 Mars, 409-413.

Kao C.H., 2000. On the differences between maximum likelihood and regression interval mapping in the analysis of quantitative trait loci. Genetics, 156, 855-865.

Le Bihan-Duval E., Berri C., Baéza E., Millet N., Beaumont C., 2001. Estimation of the genetic parameters of meat characteristics and of their genetic correlations with growth and body composition in an experimental broiler line. Poult. Sci., 80, 839-843.

Le Bihan-Duval E., Debut M., Berri C.M. Sellier N., Santé-Lhoutellier V., Jégo Y., Beaumont C., 2008. Chicken meat quality: genetic variability and relationship with growth and muscle characteristics. BMC Genetics, sous presse.

Leclercq B., Blum J.C., Boyer J.P., 1980. Selecting broilers for low or high abdominal fat: initial observations. Brit. Poult. Sci., 21, $107-$ 113.

Le Roy P., 2001. Principes et protocoles pour la détection de QTL. In : QTL - De la détection à l'utilisation. Séminaire du département de Génétique Animale de 1'INRA. 24-26 Septembre, Batz-sur-Mer, France, 1-5.

Magdelaine P., 2006. Compétitivité et segmentation du marché de la volaille. 2 ème Edition des Jeudis de la WPSA, 23 Mars, Le Mans, France.

Milan D., Jeon J.T., Looft C., Amarger V., Robic A., Thelander M., Rogel-Gaillard C., Paul S., Iannucelli N., Rask L., Ronne H., Lundström K., Reinsch N., Gellin J., Kalm E., Le Roy P., Chardon P., Andersson L., 2000. A mutation in PRKAG3 associated with excess glycogen content in pig skeletal muscle. Science, 288, 12481251 .

Nadaf J., Gilbert H., Pitel F., Berri C.M., Feve K., Beaumont C., Duclos M.J., Vignal A., Porter T.E., Simon J., Aggrey S.E., Cogburn L.A., Le Bihan-Duval E., 2007. Identification of QTL controlling meat quality traits in an F2 cross between two chicken lines selected for either low or high growth rate. BMC Genomics, 8, 155 .

Ottini L., Marziali G., Conti A., Charlesworth A., Sorrentini V., 1996. $\alpha$ and $\beta$ isoforms of ryanodine receptor from chicken skeletal muscle are the homologues of mammalian RyR1 and RyR3. Biochem. J., 315, 207-216.

Popot J., Girard J., Drouet L., 2006. La qualité technologique de la viande de Poulet Aptitude au process type «Jambon». 2ème Edition des Jeudis de la WPSA, 23 Mars, Le Mans, France.

Ricard, F.H., 1975. Essai de sélection sur la forme de la courbe de croissance chez le poulet. Dispositif expérimental et premiers résultats. Ann. Génét. Sél. Anim., 7, 427-443.

Schadt E.E., Monks S.A., Drake T.A., Lusis A.J., Che N., Colinayo V., Ruff T.G., Milligan S.B., Lamb J.R., Cavet G., Linsley P.S., Mao M., Stoughton R.B., Friend S.H., 2003. Genetics of gene expression surveyed in maize, mouse and man. Nature, 422, 297-302.

Wong G.K., Liu B., Wang J., Zhang Y., Yang X., Zhang Z., Meng Q., Zhou J., Li D., Zhang J., Ni P., Li S., Ran L., Li H., Li R., Zheng H., Lin W., Li G., Wang X., Zhao W., Li J., Ye C., Da M., Ruan J., Zhou Y., Li Y., He X., Huang X., Tong W., Chen J., Ye J., Chen C., Wei N. et al, 2004. A genetic variation map for chicken with 2.8 million single-nucleotide polymorphisms. Nature, 432, 717-722.

\section{Résumé}

Les évolutions importantes des modes de consommation des volailles (vers davantage de produits découpés ou élaborés au détriment des carcasses entières) ont renforcé l'importance de la qualité de la viande. Il existe aujourd'hui une forte variabilité de cette qualité, qu'il convient de mieux maîtriser pour contrôler les caractéristiques finales du produit. A côté des adaptations technologiques développées par les industriels, des recherches ont été initiées pour améliorer en amont la qualité de la viande, notamment par la voie génétique. Des approches complémentaires de génomique positionnelle (recherche de QTL) et expressionnelle (étude du transcriptome musculaire) ont été initiées pour identifier les gènes impliqués dans les variations de la qualité chez le Poulet. Ces études s'appuient sur des modèles animaux, lignées expérimentales ou commerciales, qui présentent de fortes différences de croissance et de qualité. Des premiers résultats originaux ont été obtenus sur les régions chromosomiques (ou QTL) contrôlant la cinétique de chute du pH post mortem, la couleur ou les pertes en eau par exsudation du filet de poulet. Les perspectives visent maintenant à identifier les polymorphismes sous-jacents, ou à défaut des marqueurs génétiques proches, pour une utilisation en sélection. Ces futures recherches bénéficieront des progrès importants réalisés dans les technologies de typage (avec l'accès chez le Poulet aux marqueurs haut débit de type SNP), mais aussi de la mise en évidence par les études du transcriptome des gènes et voies métaboliques contribuant à l'élaboration de la qualité. 


\begin{abstract}
Combined approaches of position and expression genomics for the study of genes controlling meat quality in poultry

Important changes in the ways of consumption of poultry (towards more cuts or elaborated products to the detriment of whole carcasses) have highlighted the importance of meat quality. Besides technological adaptations, research has been developed in order to improve the quality of the meat upstream, in particular by genetics. Additional approaches of positional (QTL research) and expressional (microarrays for gene expression profiling) genomics were intended to identify the genes involved in the quality of chicken meat. These studies take advantage of animal models, either experimental or commercial lines, which exhibit great differences in growth and meat quality. First original results were obtained for the chromosomal regions (or QTL) involved in the variation of post mortem $\mathrm{pH}$ fall, colour and drip loss in the breast meat of the chicken. Further studies are now aimed at identifying the underlying polymorphisms, or closely linked genetic markers, that could be used for selection. This future research will benefit from the progress made in genotyping technologies (with the access to high-density SNP markers in the chicken) as well as from the information revealed by microarray analyses on the genes and metabolic pathways contributing to chicken meat quality.
\end{abstract}

LE BIHAN-DUVAL E., BERRI C., PITEL F., NADAF J., SIBUT V., GIGAUD V., DUCLOS M., 2008. Approches combinées de génomique positionnelle et expressionnelle pour l'étude des gènes contrôlant la qualité de la viande chez les volailles. INRA Prod. Anim., 21, 159-166. 
